\title{
Percepción de la vejez y el envejecimiento en estudiantes y profesio- nistas de enfermería. Una revisión narrativa
}

\author{
Perception of old age and aging in nursing students and professionals. A \\ narrative review
}

\author{
Kira Vanessa Mendoza-Robles ${ }^{1}$ y Cristina Flores-Bello \\ ${ }^{1}$ Alumno de la carrera de Enfermería en la FES-Zaragoza, UNAM \\ ${ }^{2}$ Profesora de tiempo completo de la Carrera de Enfermería, FES Zaragoza, UNAM.
}

\section{RESUMEN}

Introducción. Los prejuicios y estereotipos negativos sobre la vejez, envejecimiento y personas mayores, que tienen muchos estudiantes y profesionales del campo de la enfermería influyen en el tipo y la calidad de atención que brindan durante el cuidado de la salud de los pacientes de mayor edad, afectando el trato digno e igualitario. Objetivo. Presentar el estado del arte del conocimiento sobre la percepción de la vejez y el envejecimiento de los estudiantes y profesionistas de enfermería. Desarrollo. Se analizó cómo el viejismo institucional e individual puede repercutir en la salud de las personas de mayor edad. También se encontró que tanto los estudiantes como profesionistas de enfermería tienen estereotipos positivos y negativos, principalmente relacionados con el área de la salud. Conclusiones. Los estudiantes y profesionales de enfermería tienen prejuicios y estereotipos tanto positivos como negativos que afectan el tipo y calidad de la atención. Su percepción se modifica con base a la escolaridad, los conocimientos adquiridos y la práctica desarrollada con adultos mayores.

Palabras clave: Enfermería gerontológica, adulto mayor, viejismo, atención de enfermería, prejuicios y estereotipos.

\section{ABSTRACT}

Introduction. The prejudices and negative stereotypes about old age, aging and the elderly, which many students and professionals in the nursing field have, influence the type and the quality of care they provide during the older patients' health care, affecting dignified and equal treatment. Objective. To present the state of the art of knowledge about the perception nursing students and professionals have about old age and aging. Development. An analysis on how institutional and individual aging can affect older people health was carried out. This revealed that both nursing students and professionals have positive and negative stereotypes, mainly related to the health area. Conclusions. Nursing students and professionals have both positive and negative prejudices and stereotypes that affect the type and quality of care. Their perception is modified based on schooling, the knowledge acquired and the practice developed with older adults.

Keywords: Gerontological nursing, elderly, ageism, nursing care, prejudices and stereotypes.
Correspondencia: Cristina Flores-Bello

E.mail: rasguosaflores@yahoo.com.mx

Artículo recibido: 4 de septiembre de 2021

Artículo aceptado: 14 de noviembre de 2021
Mendoza-Robles KV y Flores-Bello C. Percepción de la vejez y el envejecimiento en estudiantes y profesionistas de enfermería. Una revisión narrativa. CyRS. 2021; 3(2):68-79

DOI: https://doi.org/10.22201/fesz.26831422e.2021.3.2.7 


\section{INTRODUCCIÓN}

La población de adultos mayores ha aumentado a nivel mundial, incrementándose de un $9.2 \%$ en 1990 al $11.7 \%$ en 2013 , asimismo, se proyecta que para el 2050 llegará al 16\%, aunado al incremento en la longevidad. Actualmente, la mayor proporción de personas adultas mayores se encuentra en los países desarrollados, pero en las próximas décadas será en países en desarrollo. Estos cambios demográficos tendrán implicaciones sociales y de necesidades de atención a la salud incluyendo los cuidados de enfermería. ${ }^{1,2}$ En 2020, en México había 15.4 millones de personas mayores de 60 años (12\% de la población total), y la esperanza de vida promedio al nacer fue de 75.2 años y se estima una aumento al $27.7 \%$ en el $2050 .^{3}$

El envejecimiento es un proceso gradual y adaptativo de tipo biológico, psicológico y social, consecuencia de los cambios genéticamente programados, historia, estilos de vida, ambiente y condiciones sociales a las que estuvo expuesta la persona, caracterizado por una disminución en la eficiencia del funcionamiento de los órganos y sistemas del individuo y un incremento del riesgo para adquirir enfermedades agudas y crónicas. Asimismo, la vejez hace referencia a una etapa de la vida, cuyo inicio se estable por consenso dependiendo de valores culturales. La Organización de las Naciones Unidas establece que se catalogue como viejos a las personas de 60 y más años en los países en desarrollo y de 65 y más en los países desarrollados. ${ }^{4}$

Existen diferentes creencias y actitudes de los profesionales, las instituciones y la población en general sobre las personas mayores, la vejez y el envejecimiento, algunas pueden ser negativas como asociar a la vejez con la pobreza, la enfermedad y la infelicidad, mientras que otras pueden ser positivas como el pensar que todos los viejos son sabios o amables. Por otro lado, estás creencias tienen repercusión en la salud de las personas mayores, así en como los profesionales de la salud, entre ellos las enfermeras que atienden a esta población, ${ }^{5}$ por lo que le objetivo del siguiente trabajo es presentar el estado del arte del conocimiento sobre la percepción de la vejez y el envejecimiento en estudiantes y profesionistas de enfermería.

\section{VIEJISMO Y VEJEZ}

Los estereotipos son asociaciones y atribuciones específicas a un grupo, estos pueden ser características físicas, cognitivas, emocionales, preferencias de actividades, entre otros. El prejuicio es una actitud que refleja una evaluación general de un grupo, por lo general desfavorable, que se realiza sin saber, conocer o entender algo. La discriminación es un comportamiento sesgado hacia un grupo o sus miembros. Estos se aprenden a través de la escuela, amigos, medios de comunicación y las personas con las que convivimos. ${ }^{6}$

Los prejuicios y estereotipos de la vejez, envejecimiento y los viejos, que se traduce en un rechazo y discriminación se le conoce como viejismo. En este sentido, se define como cualquier actitud, acción o estructura institucional y social que subordina a una persona o grupo por razones de edad. ${ }^{5}$ En el viejismo se asume que todos los viejos son iguales por pertenecer al mismo grupo etario. Entre los rasgos negativos, se asocia a la vejez con la enfermedad, la depresión, la discapacidad cognitiva, la falta de trabajo, la pobreza y la fealdad; entre los positivos se encuentran la sabiduría, la amabilidad y el respeto. Estos son estereotipos porque los rasgos tanto negativos como positivos, no son propios de la edad ni es una descripción que se pueda realizar a todas las personas mayores. ${ }^{7,8}$

Se piensa que el viejismo es ocasionado por miedo y rechazo a envejecer, a la incapacidad de aceptar el proceso de envejecimiento como una etapa natural de la vida; al miedo, angustia, estigmas, generalizaciones y rechazo a la idea de llegar a viejo o ser viejo. Butler y Lewis en 1977 refieren cuatro factores en la sociedad actual que favorecen este fenómeno: i) temor a la muerte (ej. el que sea un tema tabú hablar sobre este tema); ii) énfasis en la cultura de la juventud (ej. anuncios que indican que la juventud es algo deseable y la vejez algo indeseable asociado a enfermedades); iii) énfasis en la productividad (ej. se valora más la productividad que otros valores como el desarrollo personal o el cuidado); y iv) los enfoques de estudio del envejecimiento (ej. en los estudios se realiza un énfasis sobre los viejos con enfermedades). ${ }^{5}$ 
Entre algunos estereotipos negativos asociados a la vejez se encuentra el pensar que todos los viejos son enfermos, que no son productivos, son infelices y tienen demencia, algunos positivos es que todos los viejos son sabios, ${ }^{9-12}$ sin embargo, estas opiniones no corresponden con las investigaciones sobre el envejecimiento.

En algunas investigaciones sobre el estado de salud de las personas de 60 años y más se reporta un $13.5 \%$ de viejos frágiles, es decir, con una alta probabilidad de tener una enfermedad que repercuta su salud y funcionalidad física, psicología o social. ${ }^{13}$ Ese porcentaje también se puede interpretar como que hay un $86.5 \%$ de viejos que mantienen su autonomía e independencia.

En lo social, las personas que dirigen las empresas más exitosas en términos económicos en Estados Unidos de Norteamérica tienen más de 50 años. ${ }^{12}$ Por otro lado, en México el $34 \%$ de las personas con 60 años o más siguen siendo económicamente activas, ${ }^{15}$ además, hay que considerar otras actividades en las que los viejos apoyan a la sociedad como el voluntariado, cuidado de los nietos, limpieza, pago de servicios, etc. ${ }^{16}$

A nivel psicológico, las investigaciones sobre felicidad indican que la edad en que las personas son más felices es antes de los 30 años y después de los 50 años. ${ }^{17}$ También en la vejez se sigue experimento la sexualidad, la cual va más allá de la genitalidad. Por otro lado, si bien algunas capacidades cognitivas disminuyen, incluso desde los 20 años, otras continúan incrementándose después de los 70 años y en algunos procesos cognitivos, como la memoria, se pueden encontrar muchas personas de 25 y 60 años con un rendimiento similar. ${ }^{18,19}$ En el caso de la sabiduría se relaciona con la experiencia, para alcanzarla es necesario practicar una actividad al menos cuatro horas diarias por 10 años y en cada ocasión aprender algo sobre ella. Además, la sabiduría se da en un área o actividad específica, por lo que tampoco se puede considerar que sólo por ser viejo se es sabio. ${ }^{20}$

El viejismo se transmite de generación en generación, lo que conlleva a las poblaciones más jóvenes a adoptar la imagen predominantemente negativa de la vejez y del envejecimiento y a comportarse de acuerdo con esta, haciendo más difícil la erradicación de actitudes, prácticas y sentimientos viejistas. ${ }^{11,12}$

\section{Repercusiones del Viejismo en el CUIDAdo de LA SALUD}

La discriminación por edad es un determinante social de la salud. El viejismo puede afectar a nivel estructural, esto se refiere a las políticas, prácticas o procedimientos explícitos o implícitos de las instituciones sociales que discriminan a las personas mayores; también puede incluir las acciones basadas en la edad de las personas que forman parte de estas instituciones, como el personal de un hospital. A nivel individual incluye el impacto de los estereotipos negativos de la edad basados en la cultura y las autopercepciones negativas del envejecimiento en la salud de las personas mayores. ${ }^{21}$

A nivel estructural se puede negar el acceso a un procedimiento o tratamiento a partir de la edad. Por ejemplo, no se atiende el dolor, la depresión o problemas cognitivos por pensar que son inherentes a la edad, tampoco se ofrece información sobre salud sexual. ${ }^{21}$ También pueden ser excluidas de los ensayos clínicos en cardiología, medicina interna, nefrología, neurología, medicina preventiva, psiquiatría, reumatología, oncología y urología. ${ }^{22-25}$ Incluso en estudios en los que la enfermedad se presenta con mayor frecuencia en la vejez como el Parkinson. ${ }^{24}$

En el entorno laboral de los profesionales de la salud también se observa que el viejismo tiene un impacto en la salud. En una revisión sistemática se reportó que en 27 de 34 estudios se observó una asociación entre el "viejismo en los cuidados de salud" con el estado de salud y la mayor edad de los profesionistas que atendían adultos mayores. A lo anterior se agrega que los prefesionistas de mayor edad pueden recibir menos cursos de actualización y que existen políticas que fomentan una jubilación anticipada. ${ }^{21}$ También los empleadores colocan a las personas mayores en puestos de trabajo con salarios más bajos y de menor responsabilidad, a pesar de tener calificaciones similares. ${ }^{28}$

El personal de salud puede tener conductas viejistas como uso de un lenguaje despectivo, infantilización, uso de términos protectores y el paternalismo, por ejemplo, en frases como: "son como niños", "sé lo que es mejor para usted", "a ver abuelito", "madrecita" "tiene 60 pero se ve como de 30 " "es viejo por fuera pero tiene alma de niño", entre otros. También al tratar a las personas mayores de forma infantil, con uso de diminutivos y voz 
pausada como si fueran incapaces de comprender. Por otro lado, el viejismo también se manifiesta al no permitir que tomen decisiones sobre su vida, colocando un énfasis sólo en la salud o ignorando sus padecimientos asumiendo que "son normales por la edad". ${ }^{21-28}$

El viejismo individual también afecta la salud, las conductas saludables e incluso la esperanza de vida en las personas mayores. ${ }^{29}$ Las personas con viejismo pueden tener un estilo de vida poco saludable que incluye el incumplimiento de la medicación, consumo excesivo de alcohol y tabaquismo, ${ }^{30}$ experimentar más ideas suicidas, ansiedad y depresión ${ }^{31}$ incluso la discriminación predice el bajo rendimiento cognitivo $^{32}$ y una menor posibilidad de recuperarse a una discapacidad grave. ${ }^{33}$

Los profesionales de la salud no siempre son conscientes de su comportamiento viejista, por tanto, voluntaria e involuntariamente retroalimentan y propa- gan los estereotipos. Muchas instituciones de salud funcionan bajo el modelo llamado "Hospital-Escuela", albergando una gran cantidad de estudiantes para su formación profesional a través de la práctica clínica y/o pasantías, durante este entrenamiento el estudiante adoptará actitudes y valores que observa de los profesionales de salud y que sin percatarse de ello, probablemente realizará durante su práctica profesional, de ahí la importancia de identificar estas conductas y niveles de expresión, para desarrollar programas con el objetivo de generar conciencia, combatir el viejismo y asegurar que los adultos mayores sean tratados con el mismo nivel de respeto y dignidad que cualquier otro grupo de edad. ${ }^{34}$

\section{VIEJISMO EN PROFESIONALES Y ESTUDIANTES DE ENFERMERÍA}

La percepción de las profesionistas y estudiantes de Enfermería es multidimensional, está relacionada

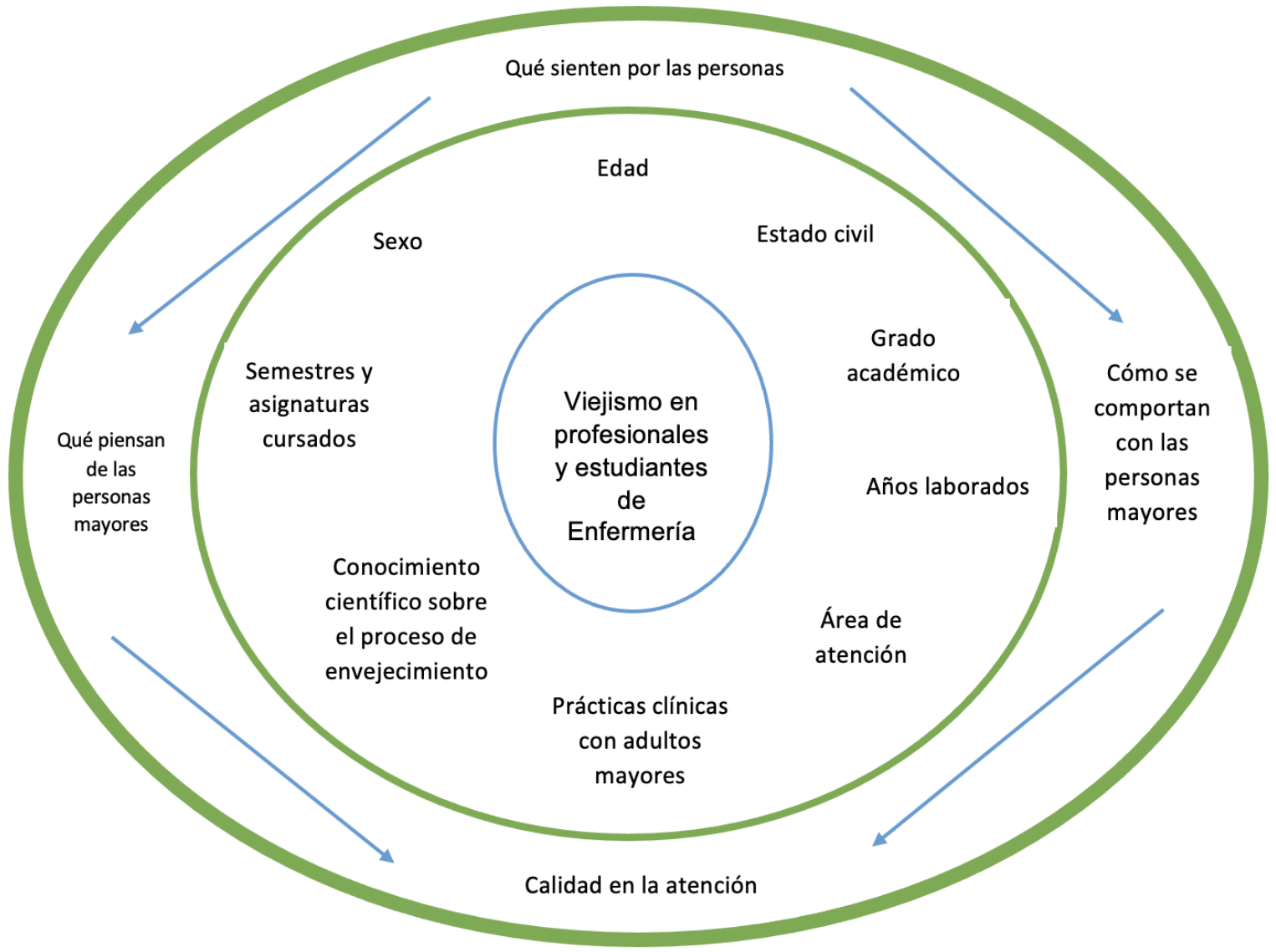

Figura 1. Factores relacionados con la percepción que tienen los profesionales y estudiantes de enfermería en relación al envejecimiento, la vejez y los adultos mayores. Los prejuicios y estereotipos están inmersos en lo cotidiano y la formación académica, lo cual tiene una influencia significativa sobre el cuidado de la salud de los adultos mayores y en muchos casos es causa de viejismo. 
con variables como la edad, el sexo y el estado civil. En profesionistas depende del grado académico, los años laborados y el área de atención donde pertenece; con respecto a los estudiantes, su percepción se modifica en relación con el semestre en que se encuentren, si han cursado alguna asignatura relacionada al cuidado del adulto mayor y a la obtención de conocimiento científico sobre el proceso de envejecimiento (Figura 1). En ambos casos el área en que existen más estereotipos sobre la vejez es la relacionada con la salud. ${ }^{36-41}$

En algunos estudios en el ámbito gerontológico y geriátrico se ha demostrado que la vejez se asocia con incapacidad física y cognitiva, fragilidad, dependencia e improductividad, lo cual repercute en la percepción y actitud ante los viejos sanos y enfermos. En este sentido, los prejuicios negativos y positivos deben ser sustituidos por la objetividad observada en las personas independientemente de la edad, ya que NO todos los viejos están enfermos, tienen deterioro cognitivo, son dependientes, incompetentes, intolerantes, rígidos, egoístas y requieren apoyos materiales, pero TAMPOCO todos son afables, agradecidos, sabios, sinceros, sociables, confiables, respetuosos, educados, cuidadosos, cariñosos y graciosos. Por tal motivo, la atención y cuidados de enfermería NO deben ser influenciados por las representaciones sociales y profesionales negativas o positivas sin fundamento, las mejores prácticas clínicas geriátricas y gerontológicas se deben sustentar en la objetividad. ${ }^{42-46}$

En un estudio sobre el viejísimo en estudiantes de enfermería se encontró una percepción moderada de viejismo en módulos donde no se revisan temas relacionados al proceso de envejecimiento o se tiene contacto con adultos mayores, mientras que los alumnos que abordaron temas relacionados al cuidado del adulto mayor la prevalencia del viejísimo disminuyó. ${ }^{47}$

La percepción del envejecimiento y vejez en estudiantes, docentes y profesionistas es conceptualizado como una fase en la vida determinada por cambios físicos y la disminución de capacidades físicas, mentales y pérdidas sociales; en cuanto a la visión positiva destacó la sabiduría, debido a la acumulación de experiencias con el paso de los años y de la reflexión sobre lo realizado en la vida, así como una etapa de desarrollo continuo pues se tiene la "opor- tunidad" para hacer aquello que de joven no se logró; se relacionó a la vejez con la soledad, el abandono, el alejarse de la familia, las pérdidas (trabajo, financiera, familia, pareja), así como ideas en torno a que el adulto mayor se vuelve lento, cansado y su muerte está muy cercana. ${ }^{49}$

Los estereotipos negativos en enfermería son producto de la constante influencia de la misma sociedad, mientras que los positivos son dados por la convivencia con un adulto mayor en casa o en el trabajo, sobre todo en aquellos enfermeros que no tuvieron esta experiencia en casa, pues el convivir con mayores sanos puede generar una percepción de comprensión, empatía y apego hacia el envejecimiento, no así, cuando se convive con ancianos enfermos y frágiles, siendo aquí cuando la percepción que se tiene de los mayores se modifica. ${ }^{48}$

La representación social de una persona o grupos de personas, se forma a partir de lo que la generalidad de una comunidad social entiende al respecto de su mundo y sus integrantes con quienes interactúa en todo momento. En este sentido, las perspectivas e imágenes que tiene la población sobre el envejecimiento provienen de una construcción social e histórica alimentada de valores y preceptos culturales, discursos políticos, sociales y posiciones de los medios de comunicación, lo cual puede variar de acuerdo con los diferentes componentes culturales que se trasmite de generación en generación en todos los espacios ya antes mencionados. ${ }^{49}$

Lo anterior es motivo de preocupación, pues los profesionales de enfermería son un elemento de especial importancia en el cuidado de las personas mayores y a la vez, de preparar no solo en conocimientos científicos y habilidades prácticas a los futuros enfermeros, sino de transmitir valores y actitudes apropiadas hacia la atención a los adultos mayores. ${ }^{36,47}$

Considerando el incremento acelerado de personas de 60 años y más en nuestro país y en consecuencia una desproporcionada demanda de atención en los siguientes años por este grupo poblacional, y partiendo de las evidencias de estudios que refieren la existencia de estereotipos negativos hacia la vejez y el envejecimiento en las enfermeras y estudiantes de enfermería (Cuadro 1), ${ }^{36-60}$ lo cual puede estar relacionado con la calidad en la atención y dar lugar a prácticas discriminatorias; resulta entonces necesa- 


\section{Cuadro 1. Estudios sobre percepción de la vejez y el envejecimiento en personal de Enfer- mería y estudiantes de la carrera de enfermería}

\begin{tabular}{llll}
\hline Autor/año & Población & Objetivos &
\end{tabular}

\begin{tabular}{|c|c|}
\hline $\begin{array}{l}\text { Medina Fer- } \\
\text { nández et al. } \\
(2021)^{36}\end{array}$ & $\begin{array}{l}212 \text { enfermeras y } \\
\text { estudiantes de Enfer- } \\
\text { maría: } \\
\text { - } 109 \text { profesionales de } \\
\text { enfermería } \\
\text { - } 10 \text { enfermeras nivel } \\
\text { técnico } \\
\text { - } 103 \text { pasantes y } \\
\text { estudiantes de enfer- } \\
\text { mería cursando entre } \\
\text { el primer y el octavo } \\
\text { semestre }\end{array}$ \\
\hline \multirow[t]{2}{*}{$\begin{array}{l}\text { Franco et al. } \\
(2010)^{47}\end{array}$} & $\begin{array}{l}200 \text { participantes, dis- } \\
\text { tribuidos de la siguien- } \\
\text { te manera: }\end{array}$ \\
\hline & $\begin{array}{l}\text { - } 80 \text { Enfermeras gene- } \\
\text { rales } \\
\text { - } 36 \text { Auxiliares de en- } \\
\text { fermería } \\
\text { - } 52 \text { Médicos adscri- } \\
\text { tos } \\
\text { - } 12 \text { Médicos residen- } \\
\text { tes } \\
\text { - } 6 \text { Médicos internos } \\
\text { - } 4 \text { Trabajadoras } \\
\text { sociales } \\
\text { - } 10 \text { Asistentes médi- } \\
\text { cas }\end{array}$ \\
\hline
\end{tabular}

Fernández et al. $(2014)^{51}$

7 profesionales de Enfermería

\begin{abstract}
Analizar la relación entre las actitudes hacia la vejez y actitudes hacia la sexualidad del adulto mayor en profesionales y estudiantes de enfermería

Se observó una relación de la edad con los estereotipos negativos físicos y, conductuales asociados a la vejez, así como relación de la edad con el miedo a envejecer, es decir, a mayor edad mayor miedo al envejecimiento, al deterioro intelectual o al abandono.
\end{abstract} de Yucatán, México.
Determinar la prevalencia de estereotipos negativos de la vejez en el personal de salud
La prevalencia de estereotipo negativo fue del $65 \%$. Una de las áreas más estereotipadas fue la relacionada a la salud del adulto mayor.
Interpretar el significado de envejecer para el profesional de enfermería en los escenarios de su práctica

Explorar la modificación de los estereotipos y mitos sobre la vejez en estudiantes del tercer grado en enfermería antes y después de impartir la asignatura "Enfermería del Envejecimiento"
El profesional de enfermería interpreta el envejecimiento como un proceso de cambios significativos, de transiciones, de pérdida de identidad y apoyo social; visualizan a los ancianos como vulnerables, en desequilibrio e incertidumbre por sus vidas tanto en el presente como en el futuro; visualizan el envejecimiento como una enfermedad y no como un proceso natural.

La prevalencia del estereotipo negativo global fue del $62 \%$ en la preintervención y del $12.3 \%$ en la postintervención. Aunque la asignatura ha demostrado ser eficaz para la modificación de estereotipos, resulta llamativo que aún algunos estudiantes mantengan niveles altos de estereotipos negativos a pesar de haber concluido la asignatura y realizado prácticas clínicas. Sugiriendo que esto se debe en parte por su propio bagaje y en parte por la influencia del sistema sanitario en el que han rotado. 
Cuadro 1. Estudios sobre percepción de la vejez y el envejecimiento en personal de Enfermería y estudiantes de la carrera de enfermería

\begin{tabular}{llll}
\hline Autor/año & Población & Objetivos & Hallazgos
\end{tabular}

\begin{tabular}{ll}
\hline Sampén- & 200 trabajadores del \\
Celis et al. & área de la salud con- \\
$(2012)^{53}$ & formado por: \\
& • 58 Médicos, \\
& • 18 Lic. en Enfermería \\
& tetricia \\
& • 9 Odontólogos \\
& - 100 Técnicos
\end{tabular}

Ortiz Saave- 235 trabajadores del dra et al. $(2013)^{54}$ servicio de Emergencia de tres hospitales:

- Médicos $45.5 \%$

- Enfermería $45.5 \%$

- Administrativos $9 \%$

Rokaia $\quad 320$ estudiantes en el Fathi et al. cuarto año académi(2019) ${ }^{55}$ co de la carrera de enfermería

BustillosLópez et al. $(2012)^{56}$

\section{4 profesionales}

vinculados al área de la salud dentro de los cuales se incluye personal de enfermería
Realizar la validación de una Escala de Actitudes hacia el adulto mayor de Kogan y determinar las actitudes hacia el adulto mayor por parte del personal de salud del primer nivel asistencial en Tumbes.

Medir la actitud hacia el adulto mayor del personal que labora en los servicios de Emergencia en hospitales de Lima

Determinar la relación de la actitud y cuidados de enfermería a un grupo de ancianos en estudiantes de pregrado de enfermería

Analizar los estereotipos sobre el envejecimiento en profesionales de atención a personas mayores.
El personal de salud tuvo una actitud positiva hacia el adulto mayor (59\%). Las personas con más edad tienen puntajes más altos de actitudes positivas, el personal de entre 41 y 50 años tienen actitudes negativas. El personal de nivel técnico mostro actitudes más positivas en contraste a los que tienen estudios universitarios.

La actitud hacia la atención del adulto mayor fue positiva en más del $90 \%$. No se encontró diferencia de actitud entre los distintos hospitales ni entre las características de los trabajadores como sexo, edad, cargo desempeñado o tiempo de labor.

La mayoría de los estudiantes de enfermería tienen falta de conocimiento sobre el cuidado de las personas mayores, lo cual tuvo una correlación significativa con su actitud vijista.

Se observó la presencia del prejuicio paternalista en virtud de creer que el adulto mayor tiene una baja competencia además de ser muy sociable.

El interés de los profesores y alumnos a las materias sobre envejecimiento es bajo, factor negativo para lograr una cultura gerontológica.
Núñoz et al. $(2007)^{57}$
148 participantes:

- 26 profesores y 122 alumnos de carreras relacionadas al área de la salud
Conocer las percepciones de los profesores y estudiantes sobre las prácticas educativas actuales en gerontología 


\section{Cuadro 1. Estudios sobre percepción de la vejez y el envejecimiento en personal de Enfer- mería y estudiantes de la carrera de enfermería}

\begin{tabular}{llll}
\hline Autor/año & Población & Objetivos & Hallazgos
\end{tabular}

\begin{tabular}{ll}
\hline López-Me- & 200 trabajadores del \\
neses et al. & área de la salud con- \\
$(2019)^{58}$ & formado por: \\
& • 58 Médicos, \\
& : 18 Lic. en Enfermería \\
& tetricia \\
& • 9 Odontólogonaría y Obs- \\
& - 100 Técnicos
\end{tabular}

Abreu y Nogueira $(2015)^{59}$ 80 alumnos de posgrado en gerontología

RuelasGonzález et al. $(2014)^{60}$
13 trabajadores del área de la salud y 12 ancianos

\begin{abstract}
Realizar la Validación de una Escala de Actitudes hacia el adulto mayor de Kogan y determinar las actitudes hacia el adulto mayor por parte del personal de salud del primer nivel asistencial en Tumbes.
\end{abstract}

Determinar las concepciones de estudiantes de posgrado en gerontología acerca del envejecimiento

\author{
Analizar la percepción \\ que el prestador de \\ servicios de salud y el \\ adulto mayor tienen \\ sobre el maltrato al an- \\ ciano en los servicios \\ públicos de salud, en \\ ciudades selecciona- \\ das de México.
}

El personal de salud tuvo una actitud positiva hacia el adulto mayor (59\%). Las personas con más edad tienen puntajes más altos de actitudes positivas, el personal de entre 41 y 50 años tienen actitudes negativas. El personal de nivel técnico mostro actitudes más positivas en contraste a los que tienen estudios universitarios.

Los conceptos positivos más representativos asociados a la vejez fueron la sabiduría, la experiencia y madurez. En cuanto a la visión negativa definen a la vejez como un estado deficitario, donde se producen pérdidas significativas e irreversibles y describen a la persona mayor como físicamente disminuido y con deterioro físico.

El maltrato contra el anciano es una práctica naturalizada por el personal de salud y por el mismo anciano. rio su identificación para posteriormente desarrollar dentro de las instituciones de salud y de las universidades programas que destierren dichos mitos y favorezcan las actitudes positivas y adecuadas en el cuidado al adulto mayor.

Finalmente, aunque no es el objetivo de la revisión, es importante señalar el viejismo relativo a la COVID-19, el cual se manifestó en todo el mundo al establecer políticas públicas discriminatorias con el supuesto interés de un cuidado especial para los viejos, considerándolos a todos como muy vulnerables, equiparando su condición a la de las personas enfermas. No obstante, se ha demostrado que la mayor letalidad por COVID-19 en los mayores de 60 años se relaciona con las desventajas socioeconómicas y no por la vejez per se, como ha sido señalado. ${ }^{62}$

\section{Conclusiones}

Los prejuicios y estereotipos de la vejez, envejecimiento y los viejos en los estudiantes y profesionistas de enfermería están determinados por las representaciones sociales y la formación académica. Por tal motivo, es necesario que los planes de estudio de enfermería incluyan contenidos, preferentemente una asignatura sobre el "viejismo y los cuidados de enfermería", con el propósito de sensibilizar y concientizar a los estudiantes y profesores sobre esta conducta y actitud que puede afectar significativamente el cuidado de las personas adultas mayores. Por otro lado, un tema pendiente son los problemas de salud emergentes como la COVID-19, en los que el viejismo también está influyendo. 


\section{Agradecimientos}

Agradecemos al Dr. José Miguel Sánchez Nieto. Profesor de asignatura nivel A de la FES Zaragoza por la revisión y edición del documento. El manuscrito fue revisado y editado en el Programa para la Investigación Bibliográfica Científica sobre Salud (PIBCIS) de la FES Zaragoza, UNAM.

\section{Referencias}

1. Organización de las Naciones Unidas. Creciendo a un ritmo menor, se espera que la población mundial alcanzará 9.700 millones en 2050 y un máximo de casi 11.000 millones alrededor de 2100: Informe de la ONU. NY: ONU 2019 [Consultado el 15 de noviembre, 2021]. Disponible en: https://population. un.org/wpp/Publications/Files/WPP2019_PressRelease_ES.pdf.

2. United Nations Department Of Economic and Social Affairs. World Population Ageing 2020 Highlights. New York;UN; 2021. Available from:

https://www.un.org/development/desa/pd/sites/www. un.org.development.desa.pd/files/undesa_pd-2020_ world_population_ageing_highlights.pdf

3. Instituto Nacional de Estadística y Geográfica (INEGI). En México somos 126014024 habitantes: censo de población y vivienda 2020. México; 2021 [cited 2021 May 14]. p. 8. Disponible en:

https://www.inegi.org.mx/contenidos/saladeprensa/ boletines/2021/EstSociodemo/ResultCenso2020_ Nal.pdf

4. Mendoza-Núñez VM. Envejecimiento y vejez. En: Mendoza-Nuñez VM, Martínez-Maldonado ML, Vargas-Guadarrama LA. Envejecimiento activo y saludable. Fundamentos y estrategias desde la gerontología comunitaria. México: FES-Zaragoza, UNAM; 2013. p.23-36.

5. Mendoza-Núñez VM. Martínez-Maldonado ML, Vargas-Guadarrama LA. Viejísimo: Prejuicios y estereotipos de la vejez. México: FES-Zaragoza, UNAM; 2008.

6. Dovidio JF., Hewstone M, Glick P, Esses VM. Prejudice, stereotyping and discrimination: Theoretical and empirical overview. En Dovidio, M. Hewstone, P. Glick, \& V.M. Esses (Eds.). The SAGE handbook of prejudice, stereotyping, and discrimination. London: Sage; 2010. p. 3-28.

7. Fernández-Jiménez $C$. Estereotipos sobre la vejez y niveles de dependencia. Geriátrika. 2003; 19(910):310-316.

8. Álvarez-Dardet SM, Cuevas-Toro AM, Pérez-PadiIla J, Lorence-Lara B. Evaluación de los estereotipos negativos hacia la vejez en jóvenes y adultos. Rev Esp Geriatr Gerontol. 2016; 51:323-328.

9. Sanhueza J. Estereotipos sociales sobre la vejez en estudiantes mayores: un estudio de caso. RIEJS. 2014; 3(1): 217-229.

10. Gil-Barreiro M, Trujillo-Grás O. Estereotipos hacia los ancianos. Rev Cubana Med Gen Integr. 1997; 13(1): 34-38.

11. Gómez-Carroza T, León del Barco B, RabazoMéndez MJ, Castro FV. Componentes de la autopercepción de los ancianos en Extremadura. International Journal of Developmental and Educational Psychology. 2006; 2(1): 315-324.

12. Osorio-Pérez O. Envejecimiento poblacional: discriminación y políticas públicas integrales. Revista de ciencias sociales y humanidades. 2016;37(81): 133-163.

13. Ofori-Asenso R, Chin KL, Mazidi M, Zomer E, Ilomaki J, Zullo AR, et al. Global Incidence of Frailty and Prefrailty Among Community-Dwelling Older Adults: A Systematic Review and Meta-analysis. JAMA Network Open. 2019; 2:e198398-e198398.

14. Salthouse T. Consequences of Age-Related Cognitive Declines. Annu Review of Psychology. 2012; 63: 201-226.

15. Instituto Nacional de Estadística y Geográfica (INEGI). INEGI. Estadísticas a propósito del día internacional de las personas de edad (10 de octubre). Comunicado de Prensa Núm 475/19 2019:1-9. Méxi- 
co; 30 de septiembre de 2019. Disponible en: https://www.inegi.org.mx/contenidos/saladeprensa/ aproposito/2019/edad2019_Nal.pdf

16. Villar F, López O, Celdrán M. La generatividad en la vejez y su relación con el bienestar: ¿Quién más contribuye es quien más se beneficia? Anales de Psicología. 2013; 29: 897-906.

17. Diener E, Oishi S, Tay L. Advances in subjective well-being research. Nature Human Behaviour 2018;2:253-260.

18. lacub R, López PH, Winzeler MO, Bourlot $\mathrm{V}$, Muro MLG de, Paz M, et al. Desarticulando las fronteras del erotismo en la vejez. Research on Ageing and Social Policy. 2020; 8: 1-24. Disponible en:

doi:10.4471/rasp.2020.4616

https://hipatiapress.com/hpjournals/index.php/rasp/ article/view/4616/2952

19. Salthouse TA. When does age-related cognitive decline begin? Neurobiology of Aging. 2009; 30: 507-514.

20. Ericsson KA, Harwell KW. Deliberate practice and proposed limits on the effects of practice on the acquisition of expert performance: why the original definition matters and recommendations for future research. Front Psychol. 2019; 10:2396. Available from: doi: 10.3389/fpsyg.2019.02396

https://www. frontiersin.org/articles/10.3389/fpsyg.2019.02396/full .

21. Chang ES, Kannoth S, Levy S, Wang SY, Lee JE, Levy BR. Global reach of ageism on older persons' health: A systematic review. PLoS One. 2020; 15(1): e0220857. Available from: doi:10.1371/journal.pone.0220857: https://journals.plos.org/plosone/ article?id=10.1371/journal. pone. 0220857

22. Kagan SH, Meléndez-Torres GJ. Ageism in nursing. Journal of Nursing Management. 2015; 23: 644-650.

23. Paeck T, Ferreira ML, Sun C, Lin CW, Tiedemann A, Maher CG. Are older adults missing from low back pain clinical trials? A systematic review and metaanalysis. Arthritis Care Res. 2014; 66: 1220- 1226.

24. Jennens RR, Giles GG, Fox RM. Increasing underrepresentation of elderly patients with advanced colorectal or non-small-cell lung cancer in chemotherapy trials. Int Med J. 2006; 36: 216-220.

25. Morse AN, Labin LC, Young SB, Aronson MP, Gurwitz JH. Exclusion of elderly women from published randomized trials of stress incontinence surgery. Obstet Gynecol. 2004; 104: 498-503.

26. Heiat A, Gross CP, Krumholz HM. Representation of the elderly, women, and minorities in heart failure clinical trials. Arch Intern Med. 2002; 162: 1682-1688.

27. Fitzsimmons PR, Blayney S, Mina-Corkill S, Scott GO. Older participants are frequently excluded from Parkinson's disease research. Parkinsonism Relat Disord. 2012; 18: 585-589.

28. Lain D. Working past 65 in the UK and the USA: segregation into 'Lopaq' occupations? Work Employ Soc. 2012; 26: 78-94.

29. Van Wicklin SA. Ageism in nursing. Plastic Surgical Nursing.2020;40(1): 20-24.

30. Westerhof GJ, Miche M, Brothers AF, et al. The influence of subjective aging on health and longevity: a meta-analysis of longitudinal data. Psychol Aging. 2014; 29: 793-802.

31. Villiers-Tuthill A, Copley A, McGee H, Morgan $\mathrm{K}$. The relationship of tobacco and alcohol use with ageing self-perceptions in older people in Ireland. BMC Public Health. 2016 Jul 22;16:627. Available from: doi: 10.1186/s12889-016-3158-y. https://bmcpublichealth.biomedcentral.com/articles/10.1186/ s12889-016-3158-y

32. Levy BR, Pilver CE, Pietrzak RH. Lower prevalence of psychiatric conditions when negative age stereotypes are resisted. Soc Sci Med. 2014; 119: $170-174$. 
33. Levy BR. Improving memory in old age through implicit self-stereotyping. J Pers Soc Psychol. 1996; 71: 1092-1107.

34. Levy BR, Slade MD, Murphy TE, Gill TM. Association between positive age stereotypes and recovery from disability in older persons. JAMA. 2012; 308: 1972-1973.

35. Dai F, Liu Y, Ju M, Yang Y. Nursing students' willingness to work in geriatric care: An integrative review. Nurs Open. 2021; 8(5): 2061-2077.

36. Medina-Fernández IA, Medina Fernández JA, Torres-Obregón R, Sosa-Cárdenas R, Chale Pool GW, Chaparro-Díaz L. Actitudes hacia la vejez y actitudes hacia la sexualidad del adulto mayor en estudiantes y profesionales de enfermería. Gerokomos. 2021; 32(1): 17-21.

37. Muñoz Cruz R. Estereotipos hacia los ancianos por parte de los jóvenes del municipio de Los Villares (Jaén). Gerokomos. 2015; 26( 1 ): 13-17.

38. Duran-Badillo T, Miranda-Posadas C, Cruz-Barrera LG, Martínez-Aguilar ML, Gutiérrez-Sánchez G, Aguilar-Hernández RM. Estereotipos negativos sobre la vejez en estudiantes universitarios de enfermería. Rev Enferm Inst Mex Seguro Soc. 2016; 24(3): 205-209.

39. Aristizábal-Vallejo N, Morales A. Salas BC, Torres AM. Estereotipos negativos hacia los adultos mayores en estudiantes universitarios. Cuadernos Hispanoamericanos de Psicología. 2009; 9(1): 35-44.

40. Molina Del Peral JA. Estereotipo hacia los ancianos. un estudio comparativo de la variable edad. Rev. de Psicol. Gral. y Aplic. 2000; 53(3):489-501.

41. Leon S, Corre-Beltran G, Giacaman AR. Negative ageing stereotypes in students and faculty members from three health science school. Gerodontology. 2015; 32:141-148.

42. Villar-Posada F. Conocimiento sobre el envejecimiento: adaptación del FAQ (Facts on Againg Quiz) y evaluación en diferentes cohortes. Rev. de Psicol. Gral. y Aplic. 2000; 53(3):523-524.
43. Larraz B, Martín Peña J. Imagen Social de la Vejez: Un acercamiento a los principales estereotipos y las repercusiones discriminatorias en las personas mayores [tesis]. España: Universidad de Zaragoza; 2013.

44. Ruíz MV, Scipioni AM, Lentini DF. Aprendizaje en la vejez e imaginario social. Fundamentos en humanidades 2008; 17(1): 221-233.

45. Blanca-Mena MJ, Sánchez-Palacios C, Trianes VM. Cuestionario de evaluación de estereotipos negativos hacia la vejez. Rev Mult Gerontol . 2005; 15(4): 212-220.

46. Orozco-Campos N, López-Hernández D. Viejismo y empoderamiento. Los prejuicios de la vejez y la visión del propio envejecimiento. Rednutrición 2016; 7(19): 245-250.

47. Franco SM, Villarreal RE, Vargas MR, Martínez GL, Galicia RL. Estereotipos negativos de la vejez en personal de salud de un Hospital de la Ciudad de Querétaro, México. Rev Méd Chile. 2010; 138(8): 988-993.

48. Izquierdo-Barrera EA. Influencia de la formación académica sobre el Viejismo en los estudiantes de la Licenciatura de Enfermería de la FES Zaragoza [tesis]. México: Facultad de Estudio Superiores Zaragoza, UNAM; 2016.

49. Guerrero-Castañeda RF, Ojeda Vargas MG. El envejecimiento desde la percepción de enfermería. Enfermería Actual en Costa Rica. 2017;32: 9ene.2017. Disponible en:

https://www.scielo.sa.cr/pdf/enfermeria/n32/14094568-enfermeria-32-00155.pdf

50. Gutiérrez Cuéllar PC. Percepciones, imágenes y opiniones sobre la vejez desde la mirada de los adultos y jóvenes en México. Espiral. 2019; 26(75): 197-223.

51. Fernández V, Guerra CA, Rondón R, Varón $M$, Gainza C, Ortega N, et al. Significado de envejecer para profesionales de enfermería en los escenarios de su práctica. Salus. 2014; 18(3): 15-20. 
52. Sarabia-Cobo C, Castanedo-Pfeiffer C. Modificación de estereotipos negativos en la vejez en estudiantes de enfermería. Gerokomos. 2015;26(1):1012.

53. Sampén-Celis JD, Varela-Pinedo LF, Díaz-Vélez C, Tello-Rodríguez T, Ortiz-Saavedra PJ. Validación de la Escala de Actitudes hacia el Adulto Mayor de Kogan y evaluación de las Actitudes hacia el adulto mayor por parte del personal de salud del primernivel asistencial. Acta Med Per. 2012; 29(3): 148-154.

54. Ortiz-Saavedra PJ, Tello-Rodríguez T, Pérez-Correa ME, Varela-Pinedo LF, Chávez-Jimeno HA. Actitudes hacia la atención del adulto mayor por parte del personal que labora en los servicios de Emergencia en hospitales de Lima. Acta méd. Peruana. 2013; 30( 2 ): 75-78.

55. Rokaia Fathi M, Alzahra Abdel AO. Conocimiento sobre el cuidado de los ancianos y su relación con la actitud del ageismo entre los estudiantes de pregrado de enfermería. Revista estadounidense de investigación en enfermería. 2019; 7 (1): 73-78.

56. Bustillos-López A, Fernández-Ballesteros R. Efecto de los estereotipos acerca de la vejez en la atención a adultos mayores. Salud Pública Méx. 2012; 54(2): 103-107.

57. Mendoza-Núñez VM, Martínez-Maldonado ML, Correa-Muñoz E. Perceptions on the importance of gerontological education by teachers and students of undergraduate health sciences. BMC Med Educ. 2007;7:1. Available from: doi: 10.1186/1472-6920-7-1.
58. López-Meneses E, Sánchez-Serrano JLS, JaénMartínez A, Gómez Galán J. Percepción de los estudiantes de posgrado en gerontología sobre el envejecimiento. Revista de Humanidades. 2019; 36: 155-180.

59. Abreu MSN, Nogueira N. Attitudes towardaging in Portuguese nursing students. Procedia - Social and Behavioral Sciences. 2015;171: 961 - 967.

60. Ruelas-González MG, Pelcastre-Villafuerte BE, Reyes-Morales $\mathrm{H}$. Maltrato institucional hacia el adulto mayor: percepciones del prestador de servicios de salud y de los ancianos. Salud pública Méx. 2014; 56(6): 631-637.

61. Bello-Chavolla OY, González-Díaz A, Antonio-ViIla NE, Fermín-Martínez CA, Márquez-Salinas A, Vargas-Vázquez $A$, et al. Unequal impact of structural health determinants and comorbidity on COVID-19 severity and lethality in older Mexican adults: considerations beyond chronological aging. J Gerontol A Biol Sci Med Sci. 2021;76(3):e52-e59. doi: 10.1093/ gerona/glaa163.

62. Hopf S, Walsh K, Flynn E, Georgantzi N. The Relationship between Ageism and Well-Being as Mediated through COVID-19-Related Experiences and Discourses. Int J Environ Res Public Health. 2021;18(19):10490. doi: 10.3390/ijerph181910490. Available: from: https://www.mdpi.com/16604601/18/19/10490 